\title{
Illusions of size change in dynamic displays
}

\author{
NICHOLAS J. WADE \\ University of Dundee, Dundee, Scotland \\ and \\ MICHAEL T. SWANSTON \\ Dundee College of Technology, Dundee, Scotland
}

\begin{abstract}
A line of constant width viewed against an expanding or contracting grating appears to become narrower or wider, respectively. This effect was studied using a computer-controlled video system with a motorized zoom lens. The magnitude of the illusory size (width) change with a vertical line did not differ when viewed against a horizontal or an oblique background, and the size change was not due to static size contrast between the line and the background. The illusory narrowing observed with an expanding background was equivalent to the widening observed with a contracting background. The apparent change in the width of lines that actually expanded was consistently underestimated, either on a similarly changing background or when viewed against a gray field. Although the transformation of the background and line produced changes in their perceived distance, this did not occur for a constant line against a transforming background, even though this condition induced the perceived size change.
\end{abstract}

Two-dimensional patterns of movement displayed on a frontoparallel surface commonly generate a perception of changing distance, which may be relative to the observer or to parts of the display itself (see Börjesson and von Hofsten, 1972; Johansson, 1964). If a complex background undergoes continuous expansion or contraction, the resulting two-dimensional transformation constitutes a flowfield of the type Gibson (1979) has proposed as a potential stimulus for perceived approach and recession. If so, observation of such transformations without any other cues to distance might be expected to influence the characteristics of superimposed patterns, such as their size or shape. The perceived size of a static shape is known to be influenced by the perceived distance of the background against which it is set (e.g., Anstis, Shopland, \& Gregory, 1961; Emmert, 1881).

One way to produce continuous optical expansion or contraction is by movement of the observer, although such transformations are difficult to control under experimental conditions. Therefore, it is preferable to simulate the transformations and present them to a stationary observer. Studies of expansion patterns as cues to distance for static observers have often involved approximations to the true transformations (e.g., Regan \& Beverley, 1979; Wallach \& Flaherty, 1975). Uniformly expanding fields, such as those generated perceptually by rotating spirals, do not reproduce the increase in motion with distance from the focus of expansion (the station point).

This research was sponsored by the Medical Research Council of Great Britain (Project Grant No. G8104633N). N. J. Wade's mailing address is: Department of Psychology, University of Dundee, Dundee DD1 4HN, Scotland.
Swanston and Wade (1981) have described a technique that employs motorized zoom lenses and video displays and produces an optical transformation in the frontoparallel plane that corresponds to movement in depth by either the observer or the surface observed. The present experiments investigated the effects of a background expansion or contraction pattern on the perceived size of a superimposed target which either shared or lacked this transformation.

\section{EXPERIMENT 1}

This experiment compared judgments of the changing size of an expanding vertical line superimposed either on an expanding horizontal background grating or against a gray field. These judgments of continuous change were compared with the difference between estimates of width obtained from viewing the static terminal points of the expansion.

\section{Method}

Subjects. Eight subjects took part in this experiment; all had normal or corrected-to-normal vision. In this and in the following experiments, the subjects were naive regarding both the method of effecting the observed transformations and the purpose of the experiment.

Apparatus. The apparatus was essentially similar to that described by Swanston and Wade (1981). It consisted of a video camera with the axis of its motorized zoom lens aligned with the center of a large horizontal square-wave grating. The superimposed line in this experiment was attached to the grating, and its length was such that the ends were not visible to the subject. The output of the camera was relayed to the subject's monitor, which was located in an adjacent darkened room. The motorized zoom lens and the electronic shutter were controlled by a microcomputer. A circular mask over the subject's monitor subtended $12 \mathrm{deg}$ at the eye, and this formed the static frame of reference 
within which the transformations were seen. The subject's head position was controlled by means of a chinrest and forehead support. The spatial frequency of the background grating was initially 2.6 cycles/deg, expanding to 1.1 cycles/deg during 2 -sec zoom-in. The line increased from $8 \mathrm{~mm}(0.8 \mathrm{deg})$ to $20 \mathrm{~mm}(2.0 \mathrm{deg})$ in width, and it was as dark as the black lines of the grating. The average luminance of the display on the monitor was $12.1 \mathrm{~cd} / \mathrm{m}^{2}$, and the Michelson contrast of the grating was 0.8 .

Procedure. The initial and final states of the display are illustrated in Figure la for the two conditions in which the background was visible. There were two modes of transition between these terminal states, and they required different judgments. In the dynamic condition, the display was visible continuously and the subjects estimated the width change, in millimeters, of the superimposed line. Prior to testing, a separation of $10 \mathrm{~mm}$ between two thin lines was shown to the subject; the lines were drawn on transparent acetate and placed directly in front of the screen. The subjects were told that the separation was $10 \mathrm{~mm}$ and that they had to judge the width change of a vertical line in millimeters. If the line appeared to expand, they signed the change positively, and if the line appeared to contract, they signed their judgments negatively. In the static condition, the display was presented unchanged for $1 \mathrm{sec}$ at the initial state, then obscured for $2 \mathrm{sec}$, during which a blank field was visible, and then presented again for $1 \mathrm{sec}$ in the final state. The subjects were required to estimate the absolute width of the line, in millimeters, at each presentation. All the trials involving either dynamic or static judgments were presented together; half the subjects commenced with the dynamic conditions and half with the static. Within a given condition, the subjects made 10 judgments, and the trials with the grating and the gray backgrounds were randomly intermixed.

\section{Results}

The static conditions yielded one width judgment for the initial and another for the final dimensions. In order to determine the static width difference, the first of these was subtracted from the second. The

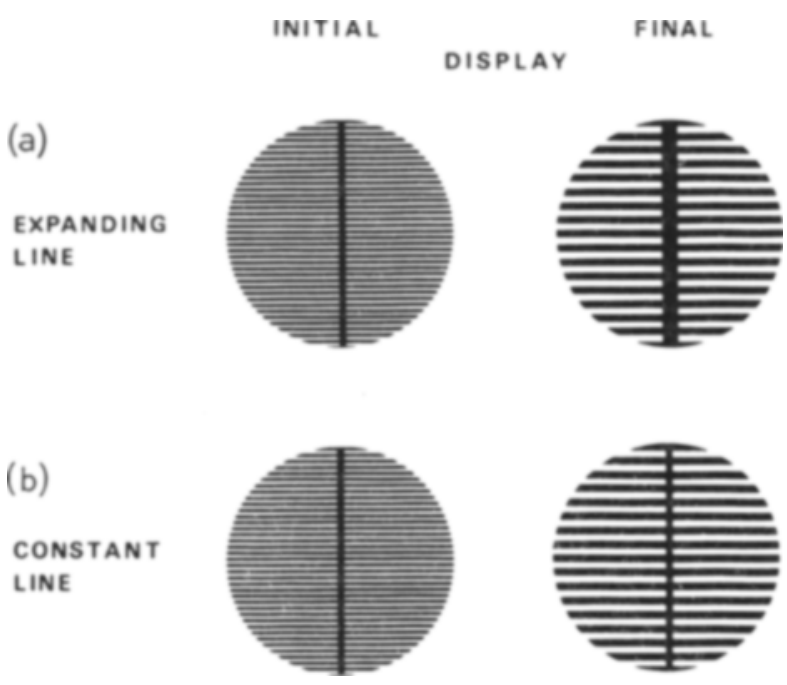

Figure 1. A diagrammatic representation of the initial and final display configurations with the expanding and constant lines. For the zoom conditions, the display expanded continuously for 2 sec between these states. For the static conditions, the displays were presented in succession for $1 \mathrm{sec}$ at each of these states. The illustration shows a horizontal background; the same conditions were examined with oblique backgrounds and vertical lines in Experiment 2.
Table 1

Mean Estimates (in Millimeters) of Vertical Line Width Change With a Horizontal Grating or a Blank Gray Background

\begin{tabular}{lcc} 
& \multicolumn{2}{c}{ Background } \\
\cline { 2 - 3 } & Grating & Gray \\
\hline $\begin{array}{l}\text { Perceived width change } \\
\text { during dynamic trans- } \\
\text { formation }\end{array}$ & 8.7 & 8.5 \\
$\begin{array}{l}\text { Difference between the } \\
\text { two static estimates }\end{array}$ & 10.9 & 11.6 \\
\hline
\end{tabular}

Note-The values given for the dynamic (zoom-in) conditions correspond to the subjects' estimates. For the static conditions the difference between two estimates, with displays equivalent to the initial and final zoom states, are given.

mean values for the derived static differences and for the width changes in the dynamic conditions are given in Table 1. From these it can be seen that the results obtained from static judgments differed from those obtained from the dynamic ones, and the difference was significant $[F(1,7)=56.18, p<.01]$. Since the dynamic judgments were derived directly and the static ones indirectly, it is possible that the measurement procedures contributed to this difference. The underestimation of width change did not, however, vary with the grating or the blank gray background $[F(1,7)=0.28, p>.05]$. There was no interaction between the effect of the type of judgment and background $[F(1,7)=1.69, p>.05]$. Thus, although a continuously perceived change in width yielded a smaller estimate of change than the difference between static judgments, the presence of an expanding background had no detectable effect. The static judgments were accurate and showed no influence of a contrast effect due to the presence of a higher or lower frequency background. While not giving evidence for a specific influence of the radial expansion on width judgments, these results provide a basis for the interpretation of Experiments 2 and 3.

\section{EXPERIMENT 2}

This experiment was designed to compare the effects of background expansion on a superimposed line that either expanded by the same amount (as in Experiment 1) or remained at a constant width. The orientation of the background grating was either horizontal or oblique, and the line was always vertical. There were therefore eight conditions in all, derived from two judgment conditions (static or dynamic), two background orientations ( 90 or $135 \mathrm{deg}$ ), and two line conditions (expanding or constant).

\section{Method}

Subjects. Sixteen subjects, all with normal or corrected-tonormal vision, took part in the experiment.

Apparatus. A line of constant width (measuring $8 \mathrm{~mm}$ on the subject's monitor) was produced electronically by a Viscount 1107 vision mixer, and it was added to the signal from the video camera. 
Table 2

Mean Estimates (in Millimeters) of the Vertical Line Width Change

\begin{tabular}{lcccc}
\hline & \multicolumn{4}{c}{ Line } \\
\cline { 2 - 5 } & \multicolumn{2}{c}{ Expanding } & \multicolumn{2}{c}{ Constant } \\
\hline $\begin{array}{l}\text { Background orientation (deg) } \\
\begin{array}{l}\text { Perceived width change } \\
\text { during dynamic transforma- }\end{array}\end{array}$ & 90 & 135 & 90 & 135 \\
$\quad \begin{array}{l}\text { tion } \\
\begin{array}{l}\text { Difference between the two } \\
\text { static estimates }\end{array}\end{array}$ & +6.3 & +6.8 & -1.9 & -2.6 \\
\hline
\end{tabular}

Note-The values given for the dynamic (zoom-in) conditions correspond to the subjects' estimates. For the static conditions the difference between two estimates, with displays equivalent to the initial and final zoom states, are given. (The + denotes apparent expansion and - signifies apparent contraction.)

Otherwise, the apparatus was the same as that employed in Experiment 1.

Procedure. Figure $1 \mathrm{~b}$ shows the initial and final states of presentation with the constant line for the horizontal background condition. The subjects were given 10 trials in each of the eight conditions, and the procedure was the same as that in Experiment 1 (without the gray field condition).

\section{Results}

As before, the static judgments were converted into estimates of width change, and the results are summarized in Table 2. The data for the expanding and constant line conditions were analyzed separately, and the former will be considered initially. The line increased in width by $12 \mathrm{~mm}$ between the terminal states, and this was underestimated in all conditions. However, the apparent size change was significantly less in the dynamic than in the static conditions $[F(1,15)=15.12, p<.01]$. This confirmed the result of Experiment 1, which had shown that such an effect was not the result of the background pattern transformed. As would then be expected, neither the orientation of the background nor its interaction influenced the judgments significantly $[F(1,15)=1.25$ and 0.06 , respectively, $p>.05$ ].

For the line of constant width, it is evident that the static judgments were relatively accurate, being $\mathbf{8 . 7}$ and $8.4 \mathrm{~mm}$ for the initial and final states. With dynamic presentation, the line appeared to become narrower by about $2 \mathrm{~mm}$. The difference between these two conditions was significant $[F(1,15)=32.33$, $p<.01]$. Neither the orientation of the background nor its interaction with the manner of judgment was significant $[F(1,15)=3.56$ and 1.21 , respectively, $\mathrm{p}>.05]$. This illusory size change is not likely to be related to the illusory line rotation that occurs with the same display transformations (see Swanston \& Wade, 1981). The apparent line rotation varies in magnitude with its orientation relative to the background, reaching a maximum at $\mathbf{4 5} \mathrm{deg}$ and declining to zero when the line and the grating are orthogonal.

Thus, an expanding background induced an illusory narrowing of a superimposed constant line, and this size change was not due to static size contrast of the line with the backgrounds of different spatial frequency. Since the results for the expanding line condition here and in Experiment 1 showed that continuous change is underestimated, it is possible that the judgments of apparent narrowing of the constant line were also underestimated.

\section{EXPERIMENT 3}

Experiment 2 showed that a constant line appears to become narrower when the background expands. This experiment was designed to determine whether the opposite effect occurs with background contraction (due to zoom-out), and if it is symmetrical with that for the equivalent expansion. Two orientations of the whole display were examined: one with the line vertical and background horizontal and the other with the line at $45 \mathrm{deg}$ and the background at $135 \mathrm{deg}$. Dynamic judgments alone were made for both constant and changing line conditions. Therefore, eight conditions were examined, derived from the combinations of constant and changing lines, expansion (zoom-in) and contraction (zoom-out) of the background, and vertical and oblique display orientations.

\section{Method}

Subjects. Sixteen subjects with normal or corrected-to-normal vision were tested.

Apparatus. The apparatus was essentially similar to that described for Experiment 2. The constant line was $14 \mathrm{~mm}$ wide in this experiment. The terminal dimensions of the changing line were 8 and $20 \mathrm{~mm}$, and the zoom duration was $2 \mathrm{sec}$. The eight conditions were prerecorded onto U-matic video tape and played back to subjects in a random order during the experiment.

Procedure. The procedure was equivalent to that for the dynamic conditions in Experiment 2. The subjects reported the apparent width change of the line, and signed positively for expansion and negatively for contraction. Each subject received $\mathbf{4 0}$ trials in all, 5 for each condition, presented in random order.

\section{Results}

The mean estimates of line width change are given in Table 3 for all eight conditions. Taking first the changing line conditions, it is clear that the width changes are equivalently underestimated for both directions of zooming. Ignoring the signs of these

Table 3

Mean Estimates (in Millimeters) of Line Width Change During Zoom-In (Expansion) and Zoom-Out (Contraction)

\begin{tabular}{lrrrr}
\hline & \multicolumn{4}{c}{ Line } \\
\cline { 2 - 5 } & \multicolumn{2}{c}{ Varying } & \multicolumn{1}{c}{ Constant } \\
\hline Background orientation (deg) & 90 & 135 & 90 & 135 \\
Line orientation (deg) & 0 & 45 & 0 & 45 \\
Zoom-in (expansion) & +7.4 & +7.3 & -1.9 & -1.6 \\
Zoom-out (contraction) & -7.5 & -7.1 & +1.8 & +1.6 \\
\hline
\end{tabular}

Note-The line width either varied by the same amount as the background or it remained physically constant. (The + denotes apparent expansion and - signifies apparent contraction.) 
data, neither zoom phase nor the display orientation significantly influenced the width estimates $[\mathrm{F}(1,15)$ $=0.06$ and 1.27 , respectively, $p>.05$ ]

Expansion of the background produced an apparent narrowing of the physically constant line, and contraction of the background produced an apparent widening of the line. While the difference between these conditions was significant $[F(1,15)=42.28$, $p<.01]$, the effects were symmetrical in magnitude. Neither the factor of display orientation nor its interaction with zoom phase were significant $[F(1,15)=$ 0.05 and 0.98 , respectively, $p>.05$ ].

\section{EXPERIMENT 4}

The results of the previous experiments could be interpreted in terms of changes in the perceived egocentric distance of the background occurring as a result of the optical transformation. If this was the case, then the apparent size change of the superimposed static line could have been due to eliciting the compensatory processes responsible for size constancy. Although the transformation of the background was optically equivalent to actual approach or recession, this does not of itself imply that subjects perceived the surface in this way. The physical pattern was open to alternative perceptual interpretations, for instance that of a "rubber sheet" being distorted in the frontoparallel plane (MacKay, 1961). In this experiment, therefore, we attempted to establish whether various transformations of the line and background are seen by subjects as movements of a stable surface in depth.

\section{Method}

Subjects. Ten subjects took part in the experiment; all had normal or corrected-to-normal vision.

Apparatus. The apparatus was the same as that used in Experiment 2.

Procedure. There were four display conditions and two judgment conditions for each one. The eight conditions were ordered randomly, with each occurring five times. The background was either expanded or contracted, and the superimposed line either changed in the same way or remained constant. For each of these four displays, the subjects were required to make a judgment of the line or of the background. The subjects were instructed to in- dicate whether the designated feature (line or background) appeared to approach, recede, or remain the same. This was done by means of a verbal report $(+,-$, or 0 , respectively) following the 2-sec trial. In order to make clear the nature of the perceptual judgment required, the preliminary instructions were to judge changes in appearance of the surface depicted on the screen, so as to avoid the possibility of confusion with the distance of the screen itself.

\section{Results}

Table 4 summarizes the results of the experiment. For each condition, the values shown are the mean percentages of each report. Almost uniformly, the subjects rated the display as approaching or receding, as appropriate, when the component being judged was correspondingly transformed. There were no difficulties in making these judgments, and the perceptual experience of an approaching or receding surface was reported to be direct and unambiguous. Thus, the experiment showed that the transformations employed in the earlier experiments were sufficient to induce changes in the perceived egocentric distance of the surface displayed.

In the two conditions in which a judgment was made of a static line, the dominant outcome was an absence of change. Next to this, the most likely percept was that the line moved in the same direction as the background; a contrasting motion was least frequent. These conditions were evidently ambiguous, and the results are accordingly difficult to interpret. Since the size change effect described above was elicited under precisely these conditions, the judgment of apparent distance must have been influenced by a number of conflicting cues.

\section{DISCUSSION}

These experiments have investigated the effects of radially expanding or contracting backgrounds on the perceived size of a superimposed figure that either shares or lacks the transformation of the background. The actual width change of an expanding or contracting line was underestimated, and this was not altered by the presence or absence of a similarly changing background. The changing line conditions in Experi-

Table 4

Percentage of Subjects' Estimates of Each Direction of Depth Change

\begin{tabular}{|c|c|c|c|c|c|}
\hline Zoom Direction & Components Transformed & Judgment of & Approach & Recede & No Change \\
\hline In & Bkgd & Line & 34 & 10 & 56 \\
\hline Out & Bkgd & Line & 16 & 10 & 74 \\
\hline In & Bkgd + Line & Line & 98 & 0 & 2 \\
\hline Out & Bkgd + Line & Line & 0 & 98 & 2 \\
\hline In & Bkgd & Bkgd & 96 & 0 & 4 \\
\hline Out & Bkgd & Bkgd & 0 & 96 & 4 \\
\hline In & Bkgd + Line & Bkgd & 100 & 0 & 0 \\
\hline Out & Bkgd + Line & Bkgd & 0 & 98 & 2 \\
\hline
\end{tabular}

Note-Judgments were made of either the line or background, when either both or the background alone were transformed either by zoom-in or zoom-out. 
ments 2 and 3 further showed that the magnitude of this underestimation remained the same with horizontal and oblique backgrounds, and with both expanding and contracting displays.

However, a line of constant width viewed against an expanding or contracting background was reported to shrink or expand, respectively. Given that the presentation was of motion on a two-dimensional surface, it could be argued that the effect was a consequence of induced movement. For example, in the studies by Duncker (1929), a luminous dot surrounded by a rectangular frame appeared to move relative to the framework even when the frame was the only physically moving component of the display. The magnitude of such an induced movement depends upon the rate of background movement in a direction opposite to the observed effect. With a display of homogeneous black and white lines, zooming in or out produces perceived movement only in a direction at right angles to the lines (Swanston \& Wade, 1981; Wallach, O'Leary, \& McMahon, 1982). Thus, a perceived contraction of a vertical line would be expected against an oblique background, since a vector of the perceived background motion lies along the horizontal axis. However, by the same argument, no effect would be expected with a horizontal background, which is seen to move only in a vertical direction. No component of this motion could induce a perceived contraction in the horizontal dimension of a vertical line.

Experiment 4 showed that the transformations applied to the line and background in previous experiments were likely to be seen as changes in their perceived egocentric distance. Thus, the perceived shrinkage of a constant line on an expanding background could have derived from its appearing to recede, by contrast with the background. However, there was no evidence for any such distance contrast effect, and therefore the size change is not likely to have been due to this cause. An expanding background appears to approach the observer, and as it does so its components necessarily increase in size, by an amount that is likely to be underestimated. A constant superimposed line undergoes a contrasting perceived size change, but not a contrasting perceived distance change. This separation of perceived size and perceived distance suggests that the use of dynamic displays can provide novel information about the nature of interactions between apparent size and distance. It remains to be seen whether similar dissociations can be found using other techniques.

\section{REFERENCES}

Anstis, S. M., Shopland, C. D., \& Gregory, R. L. (1961). Measuring visual constancy for stationary or moving objects. Nature, 191, 416-417.

Börgesson, E., \& von Hofsten, C. (1972). Spatial determinants of depth perception in two-dot motion patterns. Perception \& Psychophysics, 11, 263-268.

Duncker, K. (1929). Über induzierte Bewegung. Psychologische Forschung, 12, 180-209.

Емме RT, E. (1881). Grössenverhältnisse der Nachbilder. Klinische Monatsblatt fur Augenheilkunde, 19, 443-450.

GiBson, J. J. (1979). The ecological approach to visual perception. Boston: Houghton-Mifflin.

Johansson, G. (1964). Perception of motion and changing form. Scandinavian Journal of Psychology, 5, 181-208.

MACKAY, D. M. (1961). Interactive processes in visual perception. In W. A. Rosenblith (Ed.), Sensory communication (pp. 339355). New York: Wiley.

Regan, D., \& Beverley, K. I. (1979). Visually guided locomotion: Psychophysical evidence for a neural mechanism sensitive to flow patterns. Science, 205, 311-313.

Swanston, M. T., \& W ADE, N. J. (1981). Apparent rotation of a line superimposed upon radially expanding and contracting backgrounds. Perception, 10, 265-271.

Wallach, H., \& Flaherty, E. W. (1975). A compensation for field expansion caused by moving forward. Perception \& Psychophysics, 17, 445-449.

Wallach, H., O'Leary, A., \& McMahon, M. L. (1982). Three stimuli for visual motion perception compared. Perception \& Psychophysics, 32, 1-6.

(Manuscript received June 21, 1983;

revision accepted for publication December 12, 1983.) 